\title{
A 3-D Stability Analysis of Lee Harvey Oswald in the Backyard Photo
}

\author{
Srivamshi Pittala \\ Dartmouth College \\ Emily Whiting \\ Dartmouth College \\ Hany Farid \\ Dartmouth College
}

Follow this and additional works at: https://commons.erau.edu/jdfs|

Part of the Computer Engineering Commons, Computer Law Commons, Electrical and Computer Engineering Commons, Forensic Science and Technology Commons, and the Information Security Commons

\section{Recommended Citation}

Pittala, Srivamshi; Whiting, Emily; and Farid, Hany (2015) "A 3-D Stability Analysis of Lee Harvey Oswald in the Backyard Photo," Journal of Digital Forensics, Security and Law. Vol. 10 : No. 3 , Article 4.

DOI: https://doi.org/10.15394/jdfsl.2015.1206

Available at: https://commons.erau.edu/jdfsl/vol10/iss3/4

This Article is brought to you for free and open access by the Journals at Scholarly Commons. It has been accepted for inclusion in Journal of Digital Forensics, Security and Law by an authorized administrator of Scholarly Commons. For more information, please contact commons@erau.edu. 


\title{
A 3-D STABILITY ANALYSIS OF LEE HARVEY OSWALD IN THE BACKYARD PHOTO
}

\author{
Srivamshi Pittala, Emily Whiting, Hany Farid \\ Department of Computer Science \\ Dartmouth College \\ \{Srivamshi.Pittala.GR, Emily.JW.Whiting, Hany.Farid\}@dartmouth.edu
}

\begin{abstract}
Fifty years have passed since the assassination of U.S. President Kennedy. Despite the long passage of time, it is still argued that the famous backyard photo of Oswald, holding the same type of rifle used to assassinate the President, is a fake. These claims include, among others, that Oswald's pose in the photo is physically implausible. We describe a detailed 3-D stability analysis to determine if this claim is warranted.
\end{abstract}

Keywords: Digital Forensics

\section{INTRODUCTION}

Shortly after President John F. Kennedy's assassination on November 22nd, 1963, Lee Harvey Oswald was arrested and charged with the crime. Because Oswald was killed before his trial, we never heard from Oswald a full accounting of the details of the assassination. With so many unanswered questions of that fateful day it is not surprising that numerous theories have been proposed to fill in the missing gaps. It has long been argued, for example, that Oswald did not act alone, but rather was part of a larger criminal conspiracy involving a variety of government, international, or criminal groups. These theories point to purported inconsistencies in the events of November 22nd and in the evidence collected against Oswald. One such example is a photograph ${ }^{1}$ of Oswald in his backyard (States, 1964) holstering a pistol and holding a rifle in one hand and Marxist newspapers in the other, Figure 1(a).

This photo was particularly damning because it showed Oswald holding the same type of rifle

\footnotetext{
${ }^{1}$ Although there are three backyard photos, taken in seeming succession, we focus our analysis on only one of the backyard photos.
}

that was used to assassinate President Kennedy. At the time of his arrest, Oswald claimed that this photo was fake. In addition, it has long been argued that the lighting and shadows in the photo are physically implausible, that Oswald's facial features are inconsistent with other photos of him, that the size of the rifle is inconsistent with the known length of that type of rifle, and that Oswald's pose is physically implausible (it appears as if he is standing off balance). The Warren Commission (States, 1964) and the House Select Committee on Assassinations (States, 1979) investigated claims of photo tampering and concluded that they were unwarranted. More recent studies have also refuted the claim that the lighting and shadows in the photo are inconsistent (Farid, 2009a, 2010). This earlier work, however, did not address the claims that Oswald's pose is physically implausible. We describe the construction of a physically plausible 3-D model of Oswald that includes an anatomically-based mass distribution. This model allows to determine if Oswald's pose is physically plausible. 
(c) () This work is licensed under a Creative Commons Attribution 4.0 International License

2. RELATED WORK

The past decade has seen the development of many different and complementary approaches to analyzing a photo for evidence of manipulation (Farid, 2009b; Rocha, Scheirer, Boult, \& Goldenstein, 2011). These techniques operate on the assumption that manipulation will disrupt some statistical, geometric, or physical property in an image. Manipulation is exposed by quantifying and detecting these perturbations. For example, format-specific analyses exploit artifacts that are introduced when a JPEG image is compressed multiple times (A. Popescu \& Farid, 2005; Mahdian \& Saic, 2009; Kee, Johnson, \& Farid, 2011). Pixel-based analyses detect low-level correlations that are introduced by cloning (Fridrich, Soukal, \& Lukas, 2003; Pan \& Lyu, 2010), re-sizing (A. C. Popescu \& Farid, 2005a), or non-linear filtering (Lin, Wang, Tang, \& Shum, 2005). Sensor-based analyses can detect inconsistencies in chromatic aberrations (Johnson \& Farid, 2006), color filter array interpolation (A. C. Popescu \& Farid, 2005b; Kirchner, 2010), or sensor noise (Chen, Fridrich, Goljan, \& Lukas, 2008; Fridrich, 2009). And, physically-based analyses can detect inconsistencies in reflections (O'Brien \& Farid, 2012), lighting (Johnson \& Farid, 2005, 2007; Kee \& Farid, 2010a; de Carvalho, Riess, Angelopoulou, Pedrini, \& de Rezende Rocha, 2013), or shadows (Zhang, Cao, Zhang, Zhu, \& Wang, 2009; Kee, O’Brien, \& Farid, 2013).

Physically-based methods are attractive because they are applicable in low quality and low resolution images. Within these physicallybased techniques, those that can analyze 3$\mathrm{D}$ scene properties are particularly attractive since they offer a richer source of information than 2-D image-based techniques. 3-D stability analysis, for example, is a fundamental operation in engineering (Hibbeler, 2010). Beyond design of mechanical systems, the notion of static balance has seen broad use in computer graphics and machine vision for analysis of 3-D geometry and scene understanding. Static equilibrium constraints have been incorporated into optimization techniques for objectives such as believability of character poses (Shi et al., 2007) and balance of 3-Dprinted prototypes (Prévost, Whiting, Lefebvre, \& Sorkine-Hornung, 2013). Interactive design applications apply static balance to architectural structures (Whiting, Ochsendorf, \& Durand, 2009; Vouga, Höbinger, Wallner, \& Pottmann, 2012), structurally-sound furniture (Umetani, Igarashi, \& Mitra, 2012), or sketch-based input (Derouet-Jourdan, BertailsDescoubes, \& Thollot, 2010). In the realm of 3 -D scene analysis, static equilibrium has been used as a prior in digital reconstruction, for example, to generate physically plausible urban scenes from images (Gupta, Efros, \& Hebert, 2010), reconstruct missing information in point cloud data (Shao et al., 2014), or infer orientation of man-made objects (Fu, Cohen-Or, Dror, \& Sheffer, 2008).

Leveraging this earlier work in 3-D stability analysis, we describe a new physically-based 3D forensic analyses for analyzing the $3-\mathrm{D}$ stability of a person or object from a single image and apply this analysis to the famous Oswald backyard photo. Digital 3-D models such as that used here have been widely used in various applications from aerospace, automotive, defense and military systems, medical systems, and product design (Duffy, 2008). Unlike lifelike mannequins, digital models offer more flexibility in adjusting the location, pose, height, proportions, and weight of the model. In addition, a digital reconstruction allows for more flexibility in adjusting the location and type of light source, as well as the type of camera.

\section{3-D MODEL}

We begin by fitting an anatomically plausible 3-D model to Oswald in the backyard photo shown in Figure 1(a). We start with a 3-D articulated human body of a generic male (www.makehuman.org) of the same height $(1.75 \mathrm{~m})$ as Oswald (States, 1964). The 3-D model of the body used for our analysis has two components: skeleton and skin. The skeleton is an armature representing major bones found in the human body (spine, hands, arms, legs, feet, 


\section{c) () This work is licensed under a Creative Commons Attribution 4.0 International License}

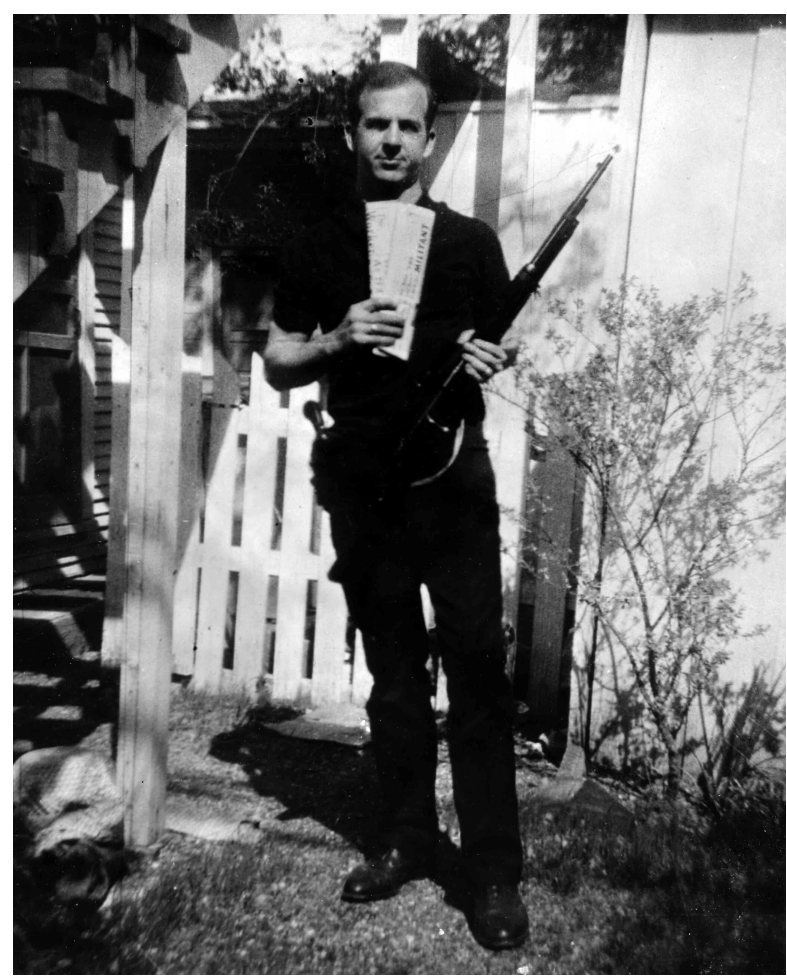

(a)

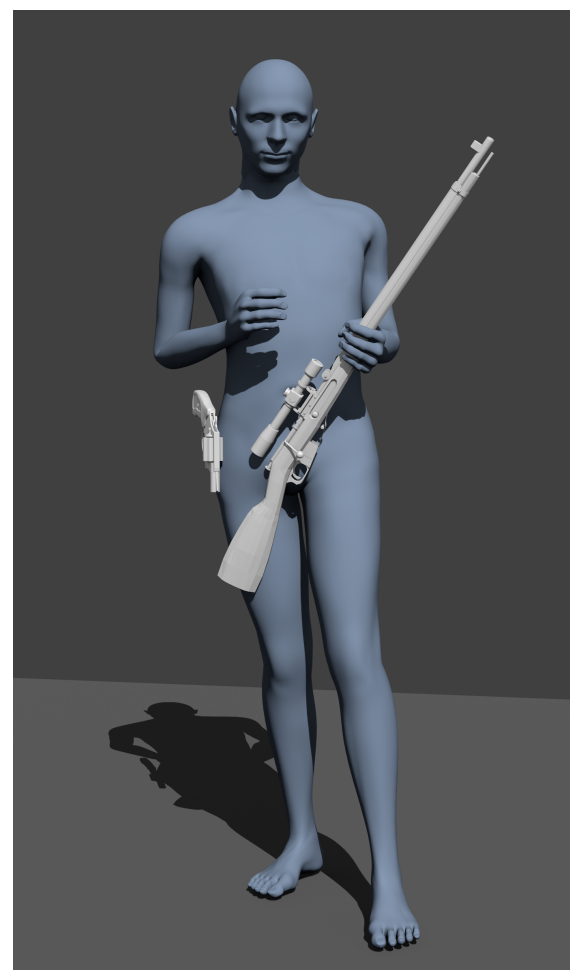

(b)

Figure 1: Shown are (a) the Oswald backyard photo and (b) a rendering of our 3-D model. etc.) The bones are connected to each other by joints and have a rotational degree of freedom about the joint. The skin is a textured mesh of triangles that overlays the skeleton. A simple colored Lambertian material is used for the skin. Although a generic 3-D head would have sufficed for our stability analysis, we employed a custom-built 3-D model generated from photographs of Oswald (Farid, 2009a).

The 3-D model can be realistically posed by simply rotating each body part about its joint. This manipulation respects the underlying physiological constraints of the human body (e.g., knees are hinge joints and an arm or leg cannot be made longer or shorter). This manipulation was performed in Blender, a freely available 3-D rendering engine. When moving a particular body part, the skin was deformed with the armature using the built in skinning tool in Blender, which applies parenting constraints to bones. The articulated model with skeleton (yellow) and skin (black) is shown in a neutral pose in Figure 3(a).

This articulated model was placed on a ground plane with the feet making direct contact with the ground. A virtual camera was created that matched the camera used to photograph Oswald - an Imperial 620 Duo Lens camera with $35 \mathrm{~mm}$ focal length and $36 \mathrm{~mm}$ sensor size (States, 1964). We manually adjusted the position and pose of the model's head, torso, arms, and legs to match Oswald's appearance in Figure 1. In the final configuration shown in Figure 1(b), the camera is $3.4 \mathrm{~m}$ from the 3 $\mathrm{D}$ model and $1 \mathrm{~m}$ above the ground plane. The matched pose is shown in Figure 3(b). Because of the inherent ambiguity in constructing a 3-D model from a single 2-D image, there are a number of 3-D geometries that are consistent with the 2-D image. The 3-D model constructed here and the subsequent analysis show that there exists a consistent and plausible $3-\mathrm{D}$ scene geometry.

Because we are interested in analyzing the 3D stability of Oswald's pose, it is necessary to add anatomically plausible masses to the skeleton. We used an anatomically-based model of human body mass distribution (DeLeva, 1996). 
(c) () This work is licensed under a Creative Commons Attribution 4.0 International License

This mass distribution model divides the human body into disjoint segments and assigns a portion of the total mass to each segment. The body segments and their corresponding share of the total body mass are: head (6.94\%), trunk (43.46\%), upper-arms $(2 \times 2.71 \%)$, forearms $(2 \times$ $1.62 \%)$, hands $(2 \times 0.61 \%)$, thighs $(2 \times 14.16 \%)$, shins $(2 \times 4.33 \%)$, and feet $(2 \times 1.37 \%)$.

We treat each segment of the body as a point mass located at its respective center of mass. This is a discrete representation for the gravitational force on each segment, where weight is applied as a single resultant force vector. By positioning a point mass at the center of mass, the discrete force vector applies a net force and net torque that is equivalent to the continuous case (Hibbeler, 2010). The point mass for each segment is assigned its share of the total mass ${ }^{2}$ of Oswald (64.4 kg) (States, 1964). The location of the center of mass for each segment was determined by a body mass distribution model (DeLeva, 1996) in which the center of mass was placed along the length of each bone. These positions for each body part are shown in Figure 2 and Figure 3(c).

In the last step, we added a 3-D model of the rifle and pistol since the masses of these may affect Oswald's overall stability. The Warren Commission identified Oswald's rifle as a $6.5 \mathrm{~mm}$ Mannlicher-Carcano, measuring $1.02 \mathrm{~m}$ in length and $3.4 \mathrm{~kg}$ in weight (States, 1964). A 3-D model of a generic rifle was scaled to the same length and its center of mass was placed halfway along its length. Oswald's pistol, a Smith \& Wesson 0.38 caliber revolver, is $20.0 \mathrm{~cm} \times 14.0 \mathrm{~cm} \times 4.0 \mathrm{~cm}$ in size and has a mass of $1.0 \mathrm{~kg}$. A $3-\mathrm{D}$ model of a generic pistol was scaled to the same size and its center of mass was placed at the model's center of mass, thus assuming a uniform density material. The

\footnotetext{
${ }^{2}$ The Warren Commission report states that at the time of his arrest, Owald gave his weight as $63.5 \mathrm{~kg}$ (140 lbs) but on other occassions he gave weights of both $63.5 \mathrm{~kg}$ and $68 \mathrm{~kg}(150 \mathrm{lbs})$. The New Orleans police records of his arrest show a weight of $61.7 \mathrm{~kg}$ (136 lbs), and the autopsy report indicates an estimated weight of $68 \mathrm{~kg}$. Given the conflicting weights, we assume a weight of $64.4 \mathrm{~kg}$ (142 lbs) the average of the various reported weights.
}

posed model with masses and rifle and pistol is shown in Figure 3(d).

One way to validate our 3 -D model is to align the rendering of the $3-\mathrm{D}$ model with the original backyard photo. The alignment, Figure 4, shows a good agreement between our model and the photo. A second way to validate our 3-D model is to verify that the shadows cast by our model match the shadows in the original photo. To this end, we model the sunlight as a distant point light source and manually positioned the light until the shadows in our model best matched the photograph. As can be seen in Figure 4 the cast shadow on the ground plane closely matches the original photo. And, shown in the lower panels of Figure 4 is a magnified view of Oswald's head showing good agreement of the shadows under the nose, lip, and neck (see also (Farid, 2009a, 2010)).

We conclude that we have constructed an anatomically plausible and consistent 3-D model of Oswald in his backyard. This 3-D model adds more evidence contradicting the claims that the shadow and lighting and length of the rifle are physically implausible.

\section{3-D STABILITY ANALYSIS}

The stability of a 3-D model is determined by comparing the projection (along the direction of gravity) of the model's global center of mass to its base of support. If the projected center of mass is contained within the base of support, then the 3-D model is stable (Goswami, 1999).

The center of mass $\vec{c}$ for our 3-D model of Oswald is computed from a weighted sum of body segments, the rifle, and the gun:

$$
\vec{c}=\frac{\left(\sum_{i=1}^{14} m_{i} \vec{c}_{i}\right)+m_{p} \vec{c}_{p}+m_{r} \vec{c}_{r}}{\left(\sum_{i=1}^{14} m_{i}\right)+m_{p}+m_{r}}
$$

where $m_{i}$ and $\vec{c}_{i}, i \in[1,14]$, correspond to the mass and center of mass of each body segment, and $m_{p}, \vec{c}_{p}$ and $m_{r}, \vec{c}_{r}$, correspond to the mass and center of mass of the pistol and rifle, respectively. Each mass is a scalar value and each center of mass is a $3-\mathrm{D}$ point. The overall $3-\mathrm{D}$ 


\section{(c) () This work is licensed under a Creative Commons Attribution 4.0 International License.}

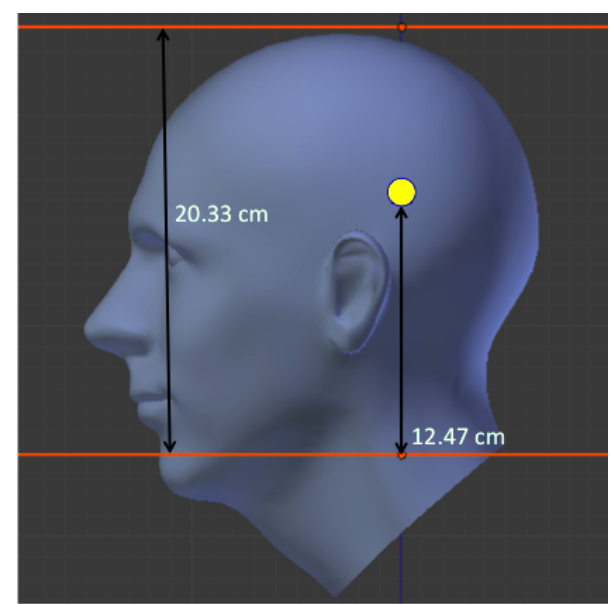

(a)

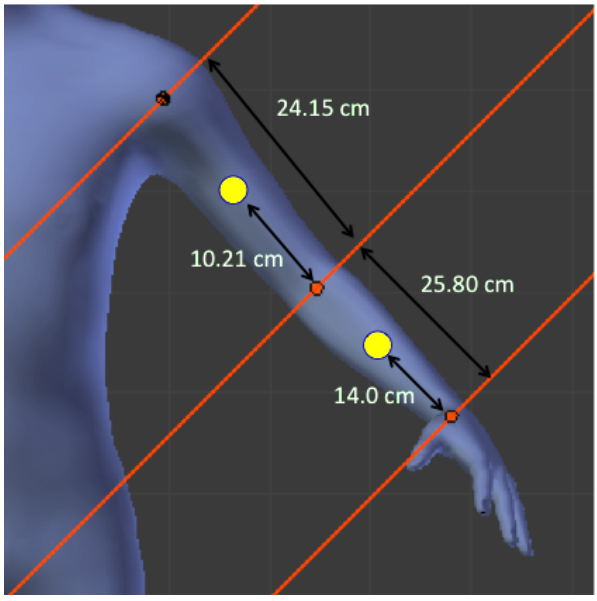

(c)

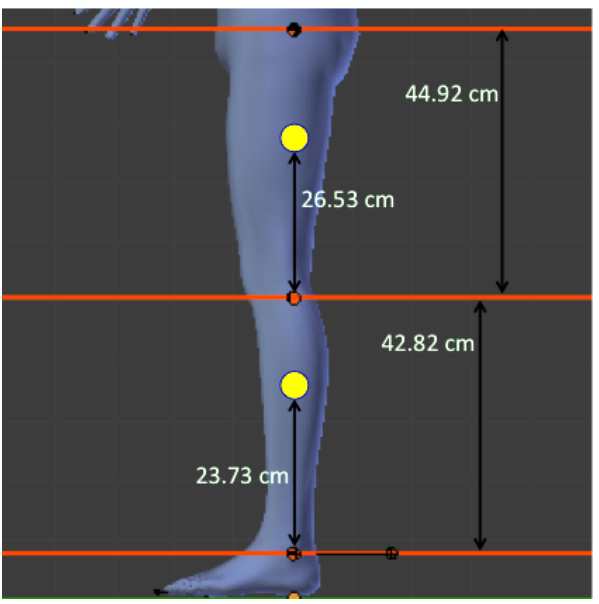

(e)

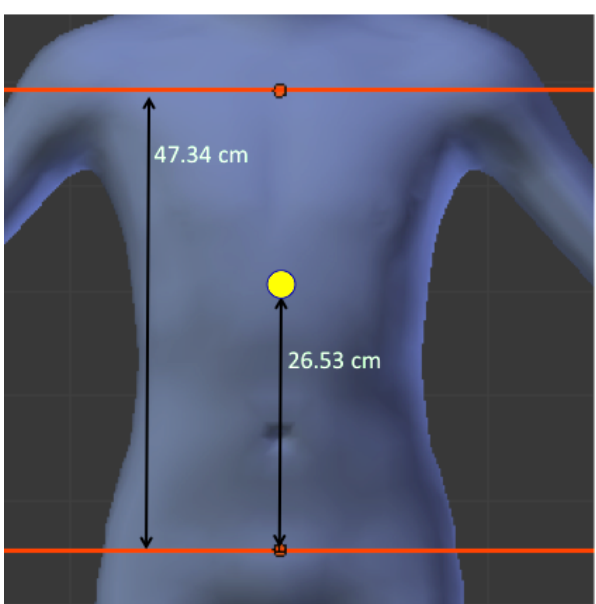

(b)

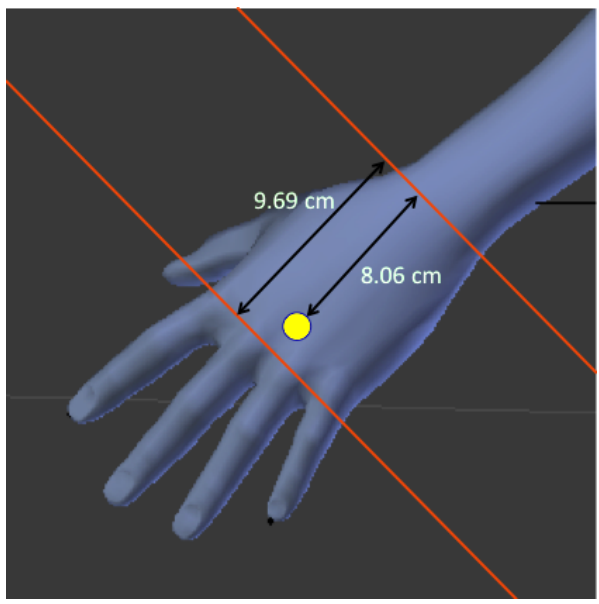

(d)

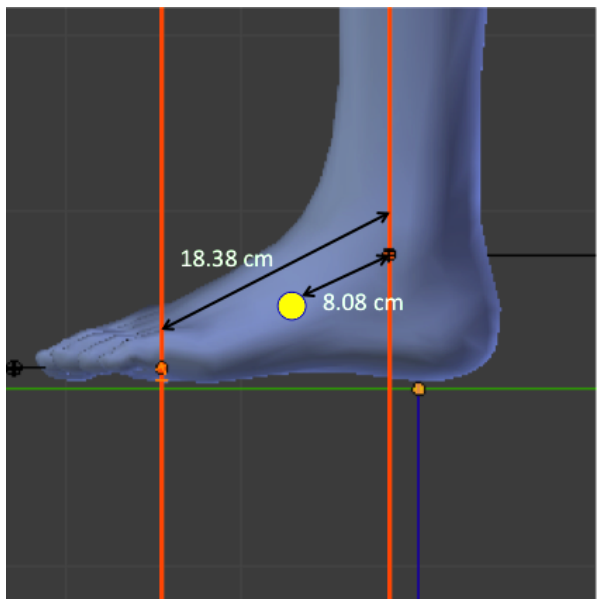

(f)

Figure 2: The relative location of the center of mass for each body segment (yellow dot). 


\section{@) (-) This work is licensed under a Creative Commons Attribution 4.0 International License}

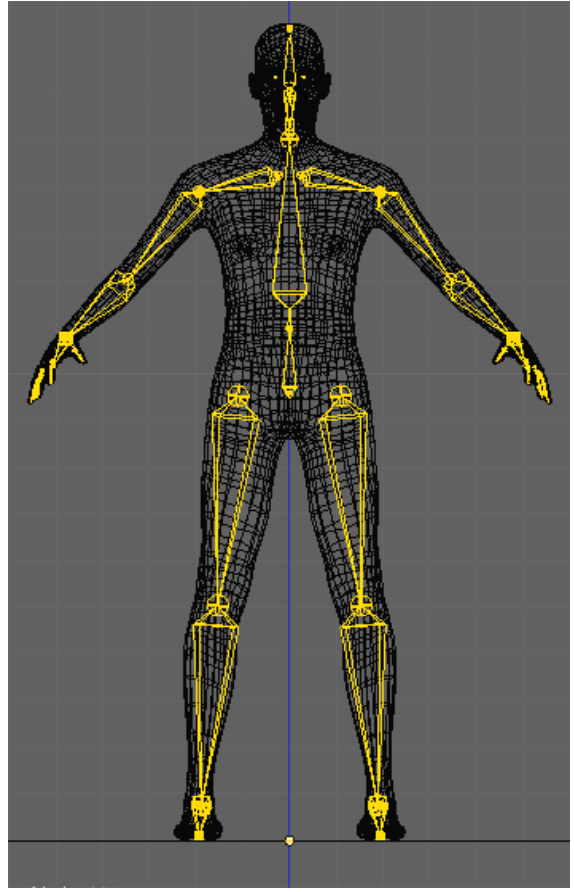

(a)

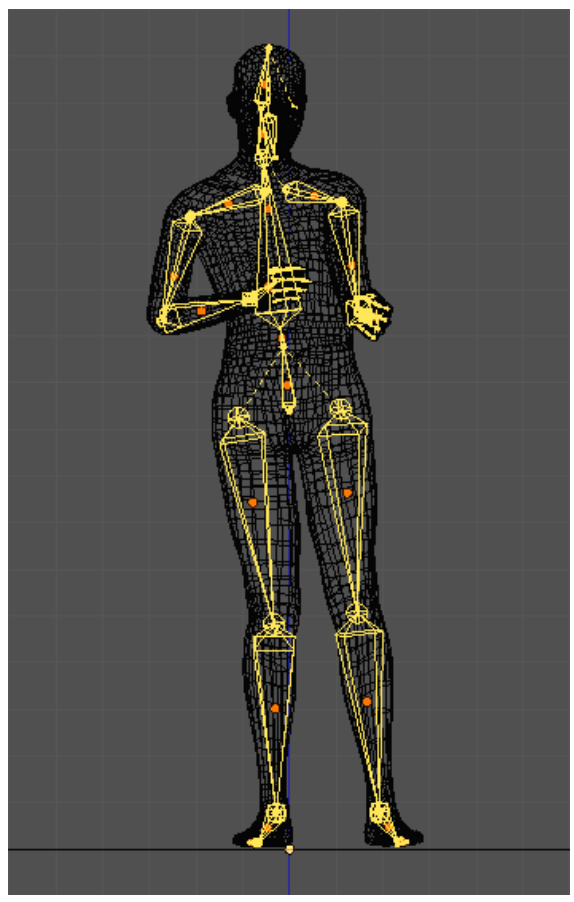

(c)

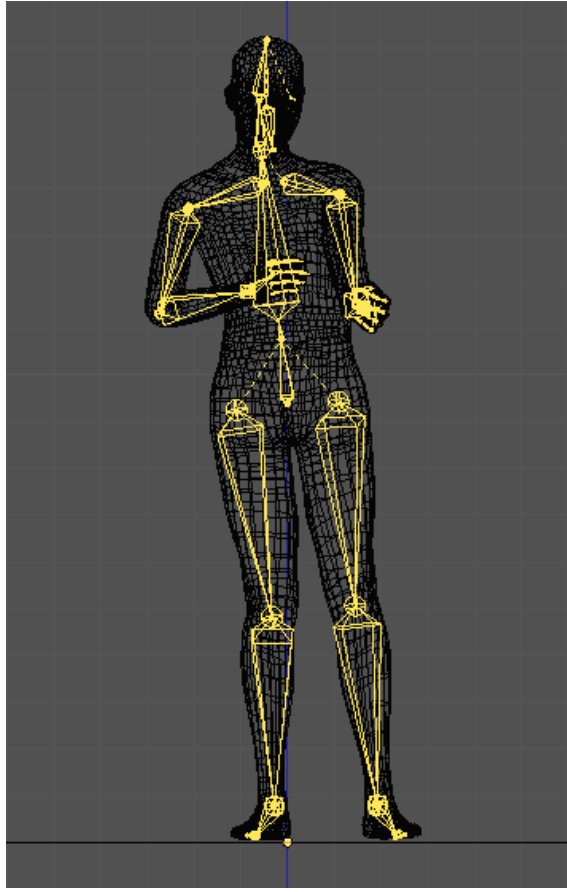

(b)

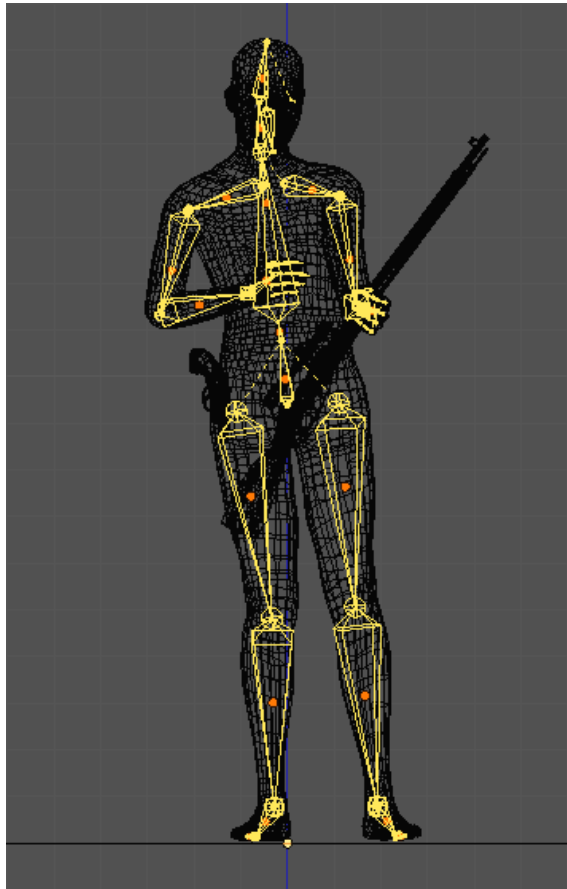

(d)

Figure 3: The 3-D articulated skeleton in a neutral pose (a), adjusted to match Oswald's pose (b), with the addition of masses (red dots) (c), and with the addition of a 3-D model of the rifle and pistol (d). 
(a) (i) This work is licensed under a Creative Commons Attribution 4.0 International License
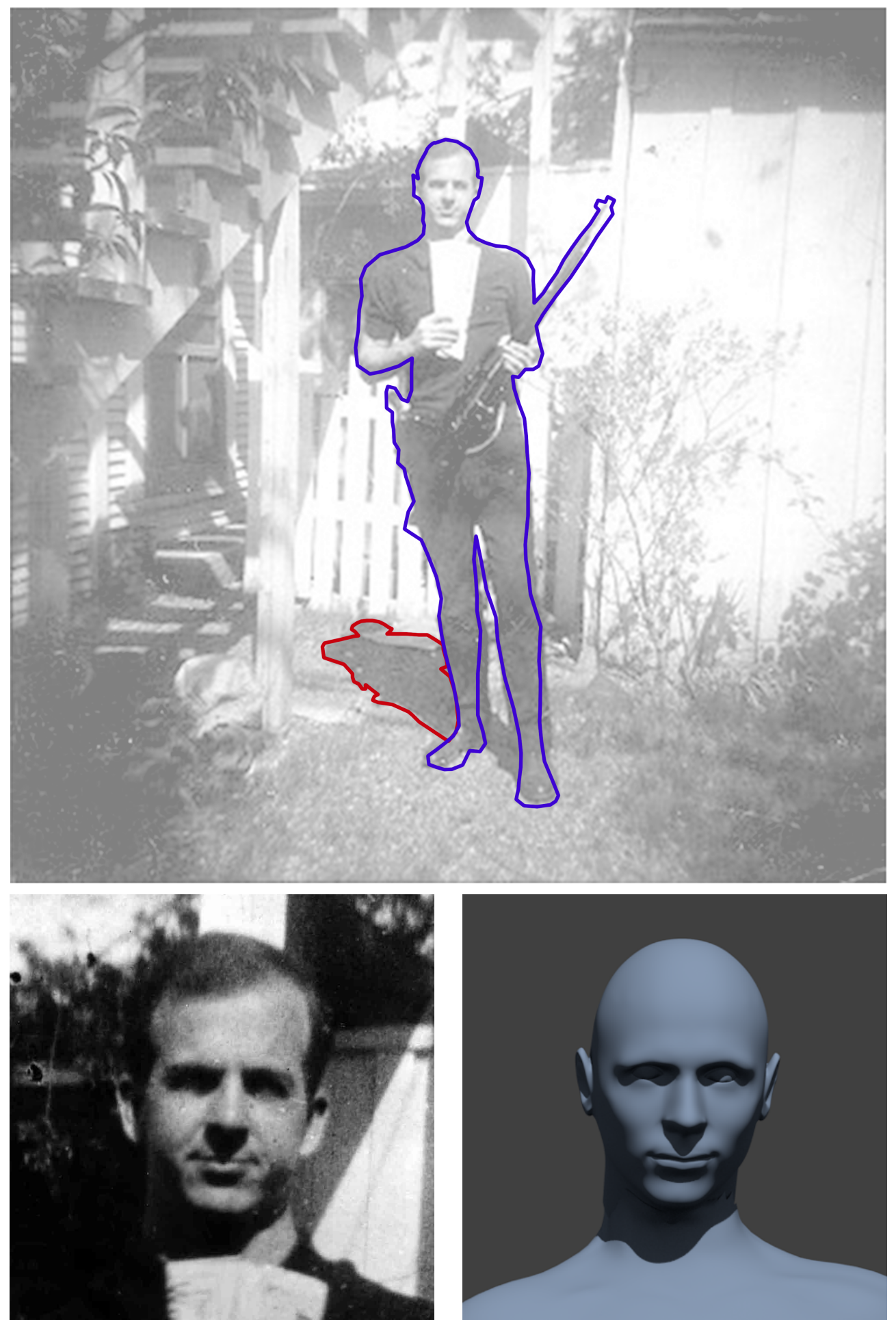

Figure 4: A superposition of the outline of our 3-D model rendering and the original backyard photo. The shadow is shown in red and the body is shown in blue. Shown in the lower panels is a magnified view of Oswald's head revealing similar shadows under the nose, lip, and neck. 
c) () This work is licensed under a Creative Commons Attribution 4.0 International License.

center of mass $\vec{c}$ is projected onto the ground plane in the direction of gravity (i.e., along $z$ axis defined to be the axis perpendicular to the ground plane). Shown in Figure 5(a) is the 3-D center of mass, $\vec{c}$, and its projection onto the ground plane, $\tilde{\vec{c}}$.

The base of support is computed as the convex hull of all points on the 3-D model that are in contact with the ground. Since we assumed that the ground is a plane, the convex hull is a 2-D polygon. A polygon is a convex hull if it fully contains the line segments between any two points within it (de Berg, Cheong, van Kreveld, \& Overmars, 2008). The base of support is shown in Figure 5(b) along with the 2-D projected center of mass. The projected center of mass lies well inside of the base of support, leading us to conclude that Oswald's pose is stable.

We of course made a number of assumptions and approximations in the construction of the 3-D model of Oswald. We applied a perturbation analysis to the 3 - $\mathrm{D}$ model in order to verify that our conclusion of Oswald's stability is not overly sensitive to these assumptions and approximations. This perturbation analysis consists of displacing the centers of mass, $\vec{c}_{i}, i \in[1,14], \vec{c}_{p}$, and $\vec{c}_{r}$ in Equation (1), recomputing the 3 -D center of mass $\vec{c}$, projecting $\vec{c}$ onto the ground plane, and comparing the projection $\tilde{\vec{c}}$ to the base of support. This perturbation accounts for potential inaccuracies in the position of each model part and the positioning of the point mass within each model part.

Each center of mass was displaced in a random direction and by a random amount, uniformly selected in the range $[0,10] \mathrm{cm}$. Shown in Figure 6 are the results of 1,000,000 random perturbations. Only $0.01 \%$ of the resulting perturbations led to an unstable pose (the projected center of mass falls outside of the base of support). Because the pose is stable with as much as $10 \mathrm{~cm}$ of displacement from the fitted model, we conclude that Oswald's pose is highly stable.

Given the conflicting reports of Oswald's weight (States, 1964), we repeated this analysis for a weight of $61.7 \mathrm{~kg}$ and $68 \mathrm{~kg}$. Oswald's pose remained nearly identically stable for these

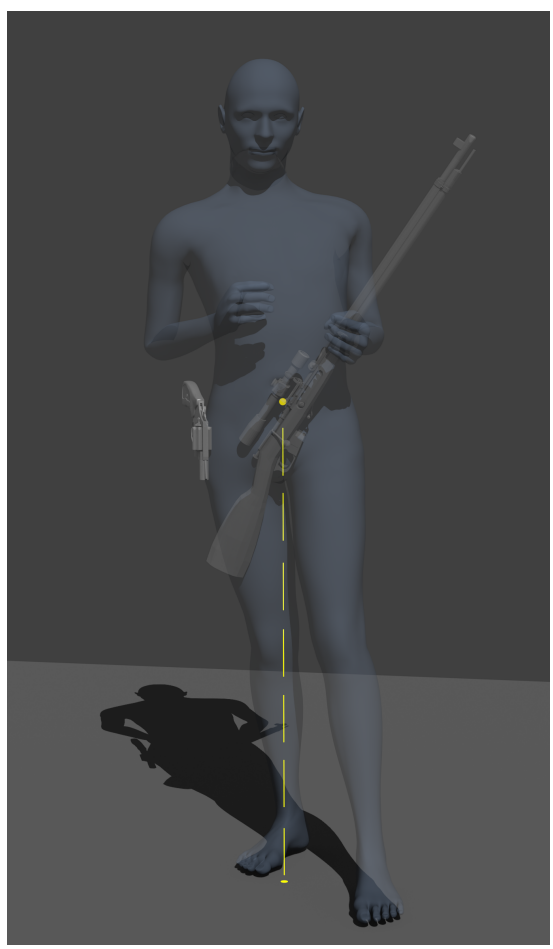

(a)

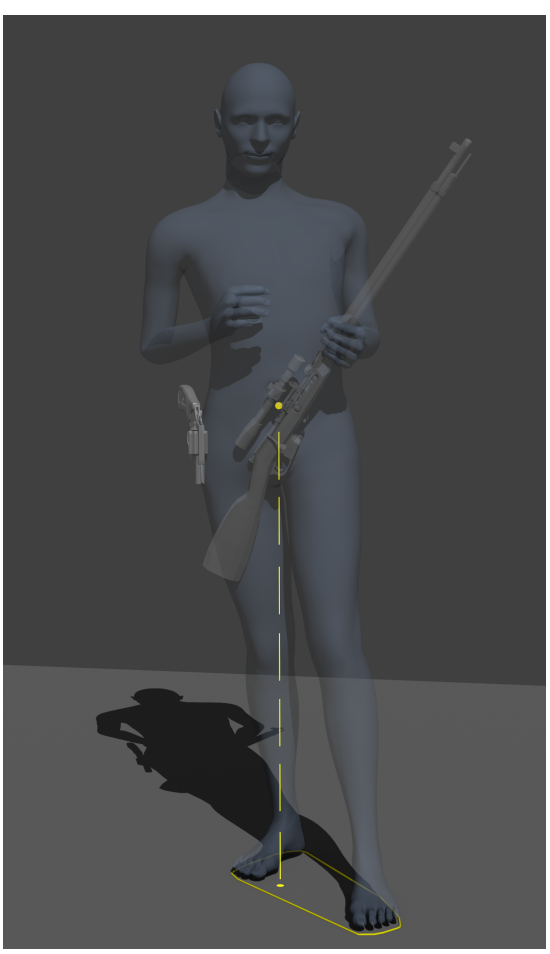

(b)

Figure 5: A 3-D view of the overall center of mass (yellow sphere) and its projection (yellow dot) onto the ground plane. The projection falls well inside the base of support (yellow polygon). 
(c) () This work is licensed under a Creative Commons Attribution 4.0 International License.
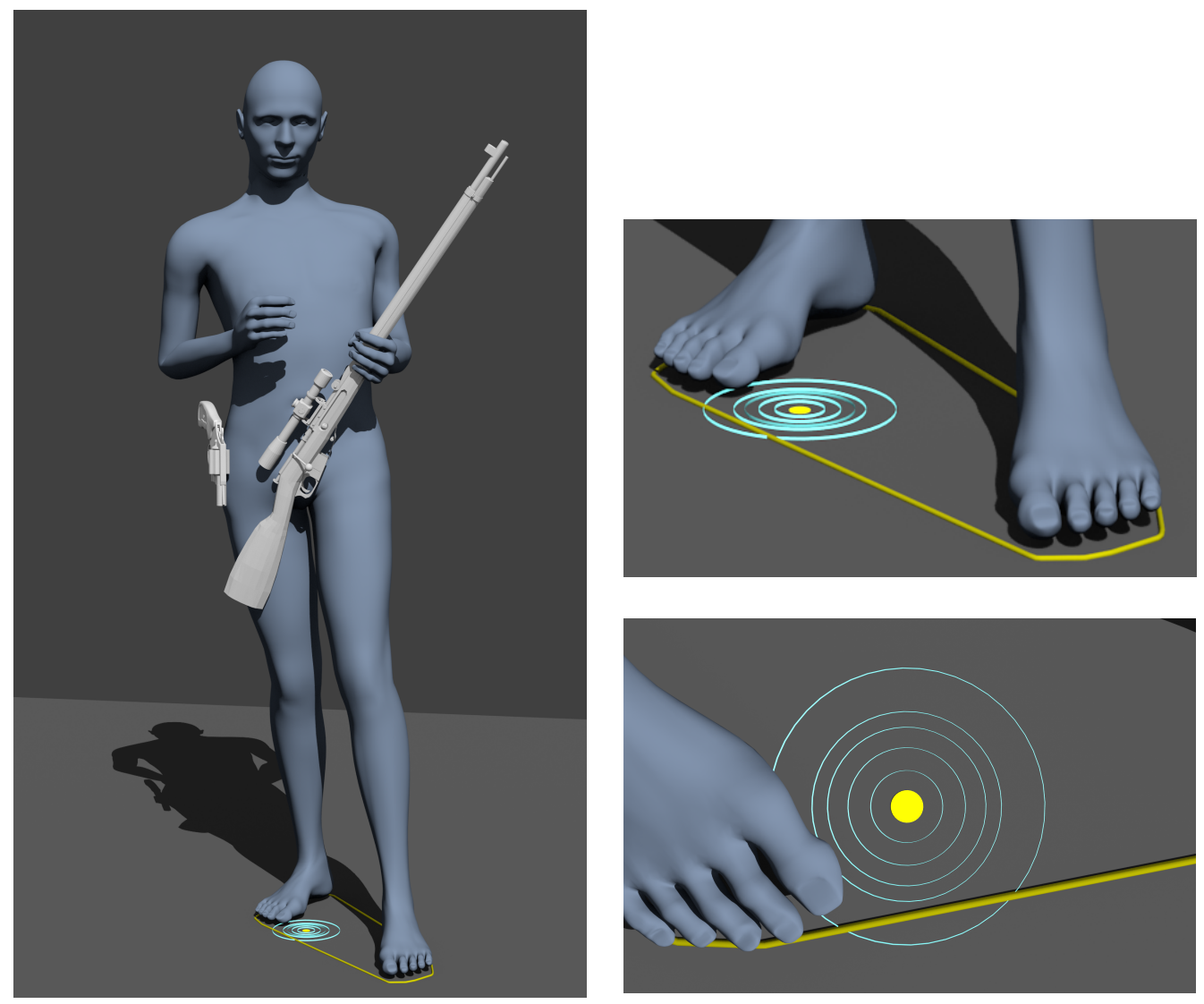

Figure 6: The yellow dot represents the projected center of mass and the yellow boundary represents the base of support. The concentric circles (cyan) represent the results of our perturbation analysis. The projected center of mass for $50 \%$ of the 1,000,000 perturbations falls within the inner most circle. The remaining circles bound $80 \%, 95 \%, 99 \%$, and $100 \%$ of the perturbed and projected centers of mass. Only $0.01 \%$ fall outside of the base of support (i.e., are unstable).

weights as for the assumed weight of $64.4 \mathrm{~kg}$.

\section{DISCUSSION}

It has long been argued that the photo of Lee Harvey Oswald in his backyard is a fake. Among other purported evidence of photo tampering, it has been argued that Oswald's pose in the photo appears physically implausible. We have described a detailed 3-D stability analysis that refutes this argument. In addition, our 3-D model of Oswald and his surroundings provide further evidence refuting other claims of photo tampering: the lighting and shadows are physically consistent, and the length of the rifle in Oswald's hands is consistent with the length of this type of rifle.

More generally, we believe that the type of detailed 3-D modeling performed here can be a powerful forensic tool in reasoning about the physical plausibility of an image. With a simple adjustment to the height and weight, the 3-D human model that we created can be used to analyze the pose, stability, and shadows in any image of people. It is possible that the manual estimation of the pose and lighting can be replaced with recent advances in automatic pose (Akhter \& Black, 2015) and lighting (Kee \& Farid, 2010b) estimation. Such advances will simplify and accelerate the speed with which 3$\mathrm{D}$ models can be constructed.

\section{REFERENCES}

Akhter, I., \& Black, M. J. (2015). Poseconditioned joint angle limits for 3D hu- 
(c) (i) This work is licensed under a Creative Commons Attribution 4.0 International License

man pose reconstruction. In IEEE Conference on Computer Vision and Pattern Recognition.

Chen, M., Fridrich, J., Goljan, M., \& Lukas, J. (2008). Determining image origin and integrity using sensor noise. IEEE Transactions on Information Forensics and Security, 3(1), 74-90.

de Berg, M., Cheong, O., van Kreveld, M., \& Overmars, M. (2008). Computational geometry: Algorithms and applications (3rd ed.). Springer.

de Carvalho, T. J., Riess, C., Angelopoulou, E., Pedrini, H., \& de Rezende Rocha, A. (2013). Exposing digital image forgeries by illumination color classification. IEEE Transactions on Information Forensics and Security, 8(7), 1182-1194.

DeLeva, P. (1996). Adjustments to ZatsiorskySeluyanovs segment inertia parameters. Journal of Biomechanics, 29(9), 12231230.

Derouet-Jourdan, A., Bertails-Descoubes, F., \& Thollot, J. (2010). Stable inverse dynamic curves. ACM Transactions on Graphics, 29(6), 137:1-137:10.

Duffy, V. G. (2008). Handbook of digital human modeling: Research for applied ergonomics and human factors engineering (1st ed.). Boca Raton, FL, USA: CRC Press, Inc.

Farid, H. (2009a). The Lee Harvey Oswald backyard photos: Real or fake? Perception, 11(38), 1731-1734.

Farid, H. (2009b). A survey of image forgery detection. IEEE Signal Processing Magazine, 2(26), 16-25.

Farid, H. (2010). A 3-D photo forensic analysis of the Lee Harvey Oswald backyard photo (Tech. Rep. No. TR2010-669). Hanover, NH, USA: Department of Computer Science, Dartmouth College.

Fridrich, J. (2009). Digital image forensic using sensor noise. IEEE Signal Processing Magazine, 26(2), 26-37.

Fridrich, J., Soukal, D., \& Lukas, J. (2003). Detection of copy move forgery in digital images. In Digital Forensic Research
Workshop.

Fu, H., Cohen-Or, D., Dror, G., \& Sheffer, A. (2008). Upright orientation of man-made objects. ACM Transactions on Graphics, $27(3)$.

Goswami, A. (1999). Postural stability of biped robots and the foot rotation indicator (FRI) point.

Gupta, A., Efros, A. A., \& Hebert, M. (2010). Blocks world revisited: Image understanding using qualitative geometry and mechanics. In European Conference on Computer Vision.

Hibbeler, R. C. (2010). Statics and mechanics of materials. Prentice Hall.

Johnson, M. K., \& Farid, H. (2005). Exposing digital forgeries by detecting inconsistencies in lighting. In 7th Workshop on Multimedia and Security (pp. 1-10). ACM.

Johnson, M. K., \& Farid, H. (2006). Exposing digital forgeries through chromatic aberration. In 8th Workshop on Multimedia and Security (pp. 48-55). ACM.

Johnson, M. K., \& Farid, H. (2007). Exposing digital forgeries in complex lighting environments. IEEE Transactions on Information Forensics and Security, 3(2), 450461.

Kee, E., \& Farid, H. (2010a). Exposing digital forgeries from 3-D lighting environments. In IEEE International Workshop on Information Forensics and Security (p. 1$6)$.

Kee, E., \& Farid, H. (2010b). Exposing digital forgeries from 3-D lighting environments. In Workshop on Information Forensics and Security.

Kee, E., Johnson, M. K., \& Farid, H. (2011). Digital image authentication from JPEG headers. IEEE Transactions on Information Forensics and Security, 6(3), 10661075.

Kee, E., O’Brien, J., \& Farid, H. (2013). Exposing photo manipulation with inconsistent shadows. ACM Transactions on Graphics, 32 (3), 28:1-28:12.

Kirchner, M. (2010). Efficient estimation of CFA pattern configuration in digital 
(c) (i) This work is licensed under a Creative Commons Attribution 4.0 International License

camera images. In Society of PhotoOptical Instrumentation Engineers Conference Series (Vol. 7541).

Lin, Z., Wang, R., Tang, X., \& Shum, H.-Y. (2005). Detecting doctored images using camera response normality and consistency. IEEE Conference on Computer Vision and Pattern Recognition, 1, 10871092.

Mahdian, B., \& Saic, S. (2009). Detecting double compressed JPEG images. In 3rd International Conference on Crime Detection and Prevention (p. 1-6).

O'Brien, J. F., \& Farid, H. (2012, January). Exposing photo manipulation with inconsistent reflections. ACM Transactions on Graphics, 31(1), 4:1-11.

Pan, X., \& Lyu, S. (2010). Region duplication detection using image feature matching. IEEE Transactions on Information Forensics and Security, 5(4), 857 -867.

Popescu, A., \& Farid, H. (2005). Statistical tools for digital forensics. In Information Hiding. Springer Berlin / Heidelberg.

Popescu, A. C., \& Farid, H. (2005a). Exposing digital forgeries by detecting traces of resampling. IEEE Transactions on Signal Processing, 53(2), 758-767.

Popescu, A. C., \& Farid, H. (2005b). Exposing digital forgeries in color filter array interpolated images. IEEE Transactions on Signal Processing, 53(10), 3948-3959.

Prévost, R., Whiting, E., Lefebvre, S., \& Sorkine-Hornung, O. (2013). Make it stand: Balancing shapes for 3D fabrication. ACM Transactions on Graphics, 32(4), 81:1-81:10.

Rocha, A., Scheirer, W., Boult, T. E., \& Goldenstein, S. (2011). Vision of the unseen: Current trends and challenges in digital image and video forensics. ACM Computing Surveys, 43(4), 26:1-26:42.

Shao, T., Monszpart, A., Zheng, Y., Koo, B., Xu, W., Zhou, K., \& Mitra, N. (2014). Imagining the unseen: Stability-based cuboid arrangements for scene understanding. ACM Transactions on Graphics, 209:1-209:11.
Shi, X., Zhou, K., Tong, Y., Desbrun, M., Bao, H., \& Guo, B. (2007). Mesh puppetry: Cascading optimization of mesh deformation with inverse kinematics. ACM Transactions on Graphics, 26(3).

States, U. (1964). The official Warren Commission report on the assassination of President John F. Kennedy. Doubleday, Garden City, N.Y.

States, U. (1979). Report of the Select Committee on Assassinations of the U.S. House of Representatives. The National Archives.

Umetani, N., Igarashi, T., \& Mitra, N. J. (2012). Guided exploration of physically valid shapes for furniture design. $A C M$ Transactions on Graphics, 31(4), 86:1$86: 11$.

Vouga, E., Höbinger, M., Wallner, J., \& Pottmann, H. (2012). Design of selfsupporting surfaces. ACM Transactions on Graphics, 31(4), 87:1-87:11.

Whiting, E., Ochsendorf, J., \& Durand, F. (2009). Procedural modeling of structurally-sound masonry buildings. ACM Transactions on Graphics, 28(5), 112:1-112:9.

Zhang, W., Cao, X., Zhang, J., Zhu, J., \& Wang, P. (2009). Detecting photographic composites using shadows. In IEEE International Conference on Multimedia and Expo (p. 1042-1045). 
(c) () This work is licensed under a Creative Commons Attribution 4.0 International License. 